\title{
An equatorial ultra iron-poor star identified in BOSS
}

\author{
C. Allende Prieto ${ }^{1,2}$, E. Fernández-Alvar ${ }^{1,2}$, D. S. Aguado ${ }^{1,2}$, J. I. González Hernández ${ }^{1,2}$, R. Rebolo ${ }^{1,2,3}$, Y. S. Lee ${ }^{4}$, \\ T. C. Beers ${ }^{5}$, C. M. Rockosi ${ }^{6}$, and J. Ge ${ }^{7}$
}

\author{
1 Instituto de Astrofísica de Canarias, Vía Láctea, 38205 La Laguna, Tenerife, Spain \\ e-mail: callende@iac.es \\ 2 Universidad de La Laguna, Departamento de Astrofísica, 38206 La Laguna, Tenerife, Spain \\ 3 Consejo Superior de Investigaciones Científicas, Spain \\ 4 Department of Astronomy and Space Science, Chungnam National University, 305-764 Daejeon, Republic of Korea \\ 5 Dept. of Physics and JINA Center for the Evolution of the Elements, University of Notre Dame, Notre Dame, IN 46556, USA \\ ${ }^{6}$ UCO/Lick Observatory, University of California, Santa Cruz, CA 95064, USA \\ 7 Department of Astronomy, University of Florida, Bryant Space Science Center, Gainesville, FL 32611-2055, USA
}

Received 15 February 2015 / Accepted 13 May 2015

\section{ABSTRACT}

\begin{abstract}
We report the discovery of SDSS J131326.89 - 001941.4, an ultra iron-poor red giant star $([\mathrm{Fe} / \mathrm{H}] \simeq-4.3)$ with a very high carbon abundance $([\mathrm{C} / \mathrm{Fe}] \simeq+2.5)$. This object is the fifth star in this rare class, and the combination of a fairly low effective temperature $\left(T_{\text {eff }} \simeq 5300 \mathrm{~K}\right)$, which enhances line absorption, with its brightness $(g=16.9)$, makes it possible to measure the abundances of calcium, carbon and iron using a low-resolution spectrum from the Sloan Digital Sky Survey. We examine the carbon and iron abundance ratios in this star and other similar objects in the light of predicted yields from metal-free massive stars, and conclude that they are consistent. By way of comparison, stars with similarly low iron abundances but lower carbon-to-iron ratios deviate from the theoretical predictions.
\end{abstract}

Key words. stars: abundances - stars: fundamental parameters - stars: Population III - Galaxy: formation - Galaxy: halo

\section{Introduction}

Only hydrogen, helium and traces of lithium were produced in the primordial nucleosynthesis, shortly after the Big Bang. The first generation of stars to form must have had the same primordial mixture of nuclei. These Population III stars started the reionization of the Universe, and created the first nuclei heavier than lithium (Truran \& Cameron 1971; Silk 1997).

At zero metallicity, gas cooling is difficult, and the formation of massive stars, with $M \gg 1 M_{\odot}$, is favored (Bromm et al. 1999; Abel \& Norman 2002). Once the metallicity of the interstellar medium reaches a level of about $10^{-4}$ times solar, cooling by fine structure lines of atomic oxygen and ionized carbon makes it much easier to form low-mass stars (Bromm \& Loeb 2003). Almost all stars with ultra-low iron abundances $([\mathrm{Fe} / \mathrm{H}]<-4.5)$ exhibit extreme carbon enhancements, which explains their formation despite their low mass (Christlieb et al. 2002; Frebel et al. 2005; Norris et al. 2007; Keller et al. 2014). However, the star recently identified by Caffau et al. $(2011,2012)$ with an iron abundance $[\mathrm{Fe} / \mathrm{H}] \simeq-4.9$, exhibits a much lower carbon abundance, suggesting that earlier predictions of the initial mass function for zero-metallicity stars need to be seriously revised (Schneider et al. 2012; Clark et al. 2013; Greif et al. 2013; Ji et al. 2014).

The chemical patterns observed in the atmospheres of ultralow metallicity stars not only illuminate the investigation of star formation at low metallicity, but, since such stars formed out of gas that may have been polluted by one or very few supernovae (Audouze \& Silk 1995), they can constrain the nucleosynthetic yields of the supernovae that ended the lives of the first stars. As of this writing, decades of search have only identified about a dozen stars with $[\mathrm{Fe} / \mathrm{H}]<-4$ (see Sect. 4). In this paper, we report on the discovery of an additional specimen in this category, currently one of the tenth most iron-poor stars known.

\section{Observations}

We identified SDSS J131326.89 - 001941.4 (hereafter J1313-0019) in observations of the Baryonic Oscillations Spectroscopic Survey (BOSS; Eisenstein et al. 2011; Dawson et al. 2013) obtained in March 2014 with the Sloan $2.5 \mathrm{~m}$ telescope (Gunn et al. 2006) and its optical spectrographs (Smee et al. 2013), and released in DR12, the final data release of the Sloan Digital Sky Survey III (SDSS; Alam et al. 2015). The BOSS spectrum of this star, bright for SDSS standards $(u=17.92, g=16.87, r=16.41, i=16.18, z=16.10)$, was obtained in the next-to-last plate of the survey. This plate (7456) and a few others, were targeted with the purpose of cross-calibrating BOSS stellar observations with those from the Sloan Extension for Galactic Understanding and Exploration (SEGUE; Yanny et al. 2009), obtained with the previous version of the SDSS spectrographs, and the Gaia-ESO survey (Gilmore et al. 2012).

The star had been observed previously as part of SEGUE (plug-plate 2901), but that spectrum was of a lower quality than the BOSS spectrum, with a median signal-to-noise ratio per pixel $^{1}$ between 5000 and $8000 \AA$ of about 40. The BOSS spectrum has an average signal-to-noise ratio per pixel of about 60 , and enables a fairly detailed analysis.

J1313-0019 was targeted by SEGUE as a metal-poor star candidate. In addition to having a lower signal-to-noise ratio, the

1 A pixel in the extracted and resampled SDSS spectra spans $69 \mathrm{~km} \mathrm{~s}^{-1}$ or $1 \AA$ at $4500 \AA$. 
SEGUE spectrum shows some systematic residuals that made it difficult to recognize the target as an ultra metal-poor star.

\section{Analysis}

We analyzed the BOSS spectrum of J1313-0019 with the software and models described by Allende Prieto et al. (2014). Briefly, the entire spectrum between $385-919 \mathrm{~nm}$ is matched with model spectra based on classical model atmospheres. We made use of the FERRE ${ }^{2}$ code (Allende Prieto et al. 2006), which searches for the model parameters that best match the observations by interpolating in a pre-computed grid of synthetic spectra. The analysis, which assumed a micro-turbulence velocity of $2 \mathrm{~km} \mathrm{~s}^{-1}$, indicated $T_{\text {eff }}=5251 \pm 50 \mathrm{~K}, \log g=1.1 \pm 0.2$, and $[\mathrm{Fe} / \mathrm{H}]=-4.5 \pm 0.1$, where the error bars combine in quadrature the internal uncertainties of the code with those inferred from the comparison with the SEGUE Stellar Parameter Pipeline (SSPP; Lee et al. 2007 and later updates) made by Allende Prieto et al. (2014). From the analysis of the globular cluster M13 $([\mathrm{Fe} / \mathrm{H}] \simeq-1.7)$, Allende Prieto et al. detected significant systematic offsets in their inferred parameters for metalpoor low-gravity $(\log g<3)$ stars. Taking those offsets into account, the preferred parameters for J1313-0019 would be $T_{\text {eff }}=$ $5320 \pm 150 \mathrm{~K}, \log g=2 \pm 0.5$, and $[\mathrm{Fe} / \mathrm{H}]=-4.2 \pm 0.2$. This analysis adopted a heliocentric radial velocity of $242 \pm 4 \mathrm{~km} \mathrm{~s}^{-1}$, derived by the SDSS specld pipeline (Bolton et al. 2012).

The SEGUE spectrum of J1313-0019, although of a significantly worse quality than the BOSS spectrum and afflicted by some obvious artifacts, suggests $T_{\text {eff }}=5505 \pm 60 \mathrm{~K}, \log g=$ $3.9 \pm 0.3$, and $[\mathrm{Fe} / \mathrm{H}]=-4.2 \pm 0.1$. This spectrum was also analyzed by the SSPP which arrived at the following parameters: $T_{\text {eff }}=5674 \pm 122 \mathrm{~K}, \log g=2.41 \pm 0.05$, and $[\mathrm{Fe} / \mathrm{H}]=$ $-4.25 \pm 0.08^{3}$. From this spectrum, the specld pipeline measured a heliocentric radial velocity of $268 \pm 6 \mathrm{~km} \mathrm{~s}^{-1}$, and provided a second value of $269 \pm 4 \mathrm{~km} \mathrm{~s}^{-1}$ from the fitting of the data with smoothed templates from the Elodie library in the 4100-6800 range. This velocity is different from the one measured in the BOSS spectrum by $26 \mathrm{~km} \mathrm{~s}^{-1}$ or about $6 \sigma$, suggesting the star may be part of a binary system, but more data are necessary to confirm this hypothesis.

The extinction estimate in the Schlegel et al. maps in the direction of J1313-0019 is $E(B-V)=0.028 \mathrm{mag}$. Correcting the observed $(g-r)$ color of the star, adopting $A_{g}=1.19 A_{V}$ and $A r=0.87 A_{V}$ (Girardi et al. 2004) and the average Galactic relationship $A_{V}=3.1 E(B-V)$, we find $(g-r)_{0}=0.49$. Using the polynomial relationship proposed by Pinsonneault et al. (2012), we arrive at $T_{\text {eff }}=5576 \mathrm{~K}$. On the other hand, the SSPP reports an IRFM-based temperature of $5669 \pm 58 \mathrm{~K}$. Both are significantly warmer than the temperatures we derive spectroscopically from the BOSS spectrum, but closer to those from the lower quality data from SEGUE. Nevertheless, we have confidence in the effective temperature inferred from the BOSS spectrum, about $5400 \mathrm{~K}$, since it reproduces both the local continuum slope and the strength of the $\mathrm{H}$ lines in the spectrum (see Fig. 1).

The spectrum of J1313-0019 shows strong CH absorption, indicating that the star is rich in carbon. The most isolated strong Fe I lines in the spectrum are in the blends around 3820 and $3860 \AA$, plus several fairly strong transitions between 3600 and $3608 \AA$, but the signal-to-noise ratio of the BOSS spectrum does not allow an unambiguous detection of individual lines or

\footnotetext{
2 FERRE is available from http://hebe.as.utexas.edu/ferre

3 We adopt the values released in the ninth data release of the SDSS (DR9; Ahn et al. 2012).
}

groups of overlapping iron lines. The overall effect of Fe I absorption between 3600 and $3900 \AA$, however, is fairly significant, with dozens of features that reduce the stellar flux by a few percent. These, as a whole, can be studied with a statistical approach. We attempted to simultaneously recover the abundances of carbon, the $\alpha$-elements $(\mathrm{O}, \mathrm{Mg}, \mathrm{Si}, \mathrm{S}, \mathrm{Ca}$ and $\mathrm{Ti})$, and iron (and the rest of the metals), assuming again a microturbulence velocity of $2 \mathrm{~km} \mathrm{~s}^{-1}$, and using (as in previous cases) $\chi^{2}$ as a metric. In this analysis, which used the full BOSS spectral range (3600-10000 $\AA$ ) we arrived at $T_{\text {eff }}=5378 \pm 50 \mathrm{~K}$, $\log g=3.0 \pm 0.2,[\mathrm{C} / \mathrm{Fe}]=+2.5 \pm 0.1,[\alpha / \mathrm{Fe}]=+0.2 \pm 0.1$ and $[\mathrm{Fe} / \mathrm{H}]=-4.3 \pm 0.1$. We also processed the BOSS spectrum of J1313-0019 through a customized version of the SSPP in which algorithms that used photometric information were not used, arriving at $T_{\mathrm{eff}}=5502 \pm 62 \mathrm{~K}, \log g=2.69 \pm 0.41$, $[\mathrm{Fe} / \mathrm{H}]=-4.37 \pm 0.15$ and $[\mathrm{C} / \mathrm{Fe}]=2.68 \pm 0.10$.

Figure 1 illustrates how well the best-fit model matches the observed BOSS spectrum over several relevant spectral windows, including the wavelengths of the $\mathrm{Ca}$ II resonance lines, the $\mathrm{G}$ band $(\mathrm{CH})$, and the $\mathrm{Ca}$ II infrared triplet. The $\mathrm{H}$ Balmer lines are the most prominent transitions in the top panels, and at $4102(\mathrm{H} \delta)$ and $4341 \AA(\mathrm{H} \gamma)$ in the middle panels. Molecular $\mathrm{CH}$ absorption is responsible for most of the remaining features apparent between 3850 and $4400 \AA$, with the notable exception of the Ca II K line at $3934 \AA$ (the Ca II H line overlaps with $\mathrm{H} \epsilon$ at $3969 \AA$ ). The strong transitions in the bottom panels correspond to the Ca II triplet (8498, 8542 and $8662 \AA$ ) and (weaker) $\mathrm{H}$ Paschen lines at 8408, 8432, 8461, 8497, 8540, 8593, 8659, 8744 , and $8857 \AA$. Figure 2 shows the chi-square contours in the $[\alpha / \mathrm{Fe}]-[\mathrm{Fe} / \mathrm{H}]$ plane in the vicinity of the minimum. The contours correspond to values of the minimum plus 1,4 , 9, etc. corresponding to the 1-2-3-etc. $\sigma$ uncertainties if we are dealing with a Gaussian error distribution $(68 \%, 95 \%, 99.7 \%$, etc $)$. The reduced $\chi^{2}$ at the minimum is 1.1 .

We measured the equivalent width of the $\mathrm{Ca}$ II $\mathrm{K}$ line to be $0.64 \AA$, and using the July 2014 version of MOOG (Sneden 1971) and an appropriate ATLAS9 model atmosphere computed as in Mészáros et al. (2012), we found that such line strength correspons to $[\mathrm{Ca} / \mathrm{H}]=-4.0$. We note in passing that an interstellar component is apparent in the Ca II resonance lines, blue-shifted by about $350 \mathrm{~km} \mathrm{~s}^{-1}$, and which is apparent in the observation and not the stellar model shown in the top right-hand panel of Fig. 1. The infrared Ca II lines are blended with Paschen lines, thus an equivalent-width analysis to estimate the $\mathrm{Ca}$ abundance would require modelling the contribution of the hydrogen lines (see Fig. 1). By adopting the atmospheric parameters obtained from the global fit and minimizing $\chi^{2}$ over the windows that are most sensitive to iron lines, as described in Fernández-Alvar et al. (2015), we arrive at $[\mathrm{Fe} / \mathrm{H}]=-4.4 \pm 0.3$. All these checks give consistent results.

We do not directly detect lines of barium or magnesium; only upper limits can be given. The $\alpha$-element abundance is constrained mainly by the Ca II resonance lines at $3950 \AA$ and the Ca II infrared triplet at 8450-8600 ̊. Examining the spectrum in the vicinity of the Ba II resonance line at $4554 \AA$ we estimate $[\mathrm{Ba} / \mathrm{Fe}]<+1.5$, and from an analysis of the region around the $\mathrm{Mg} \mathrm{I} b$ lines we estimate $[\mathrm{Mg} / \mathrm{Fe}]<+0.5$.

\section{Discussion and conclusions}

J1313-0019 is a new example of the class of stars with extremely low iron abundances and very high $\mathrm{C} / \mathrm{Fe}$ ratios. This class includes about half of the stars with $[\mathrm{Fe} / \mathrm{H}]<-4$. 


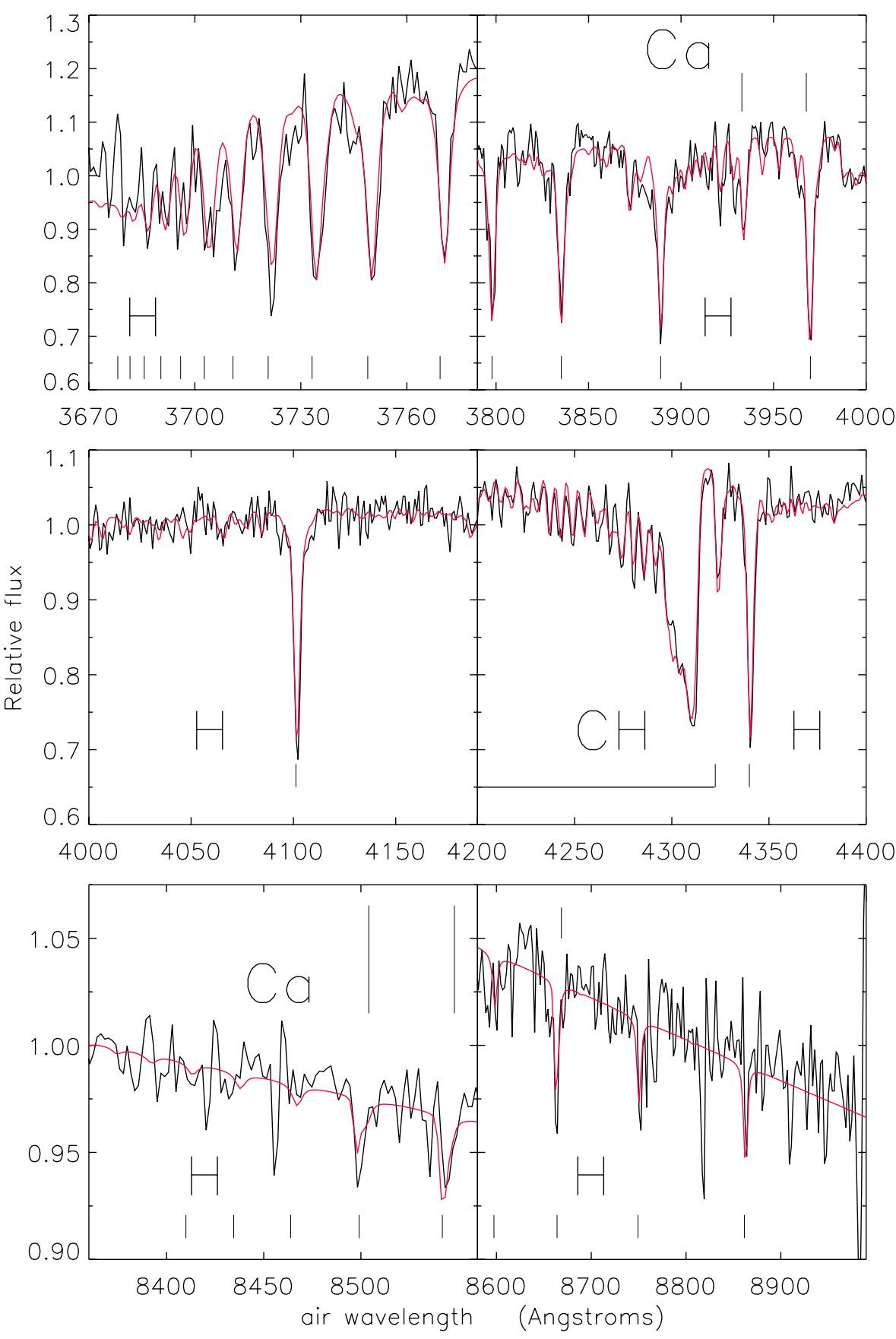

Fig. 1. BOSS spectrum of J1313-0019 (black) and best-fitting model (red). The elements or molecules associated with the most prominent lines are identified. The continuum correction has been carried out following the procedure described by Allende Prieto et al. (2014), namely dividing the spectral range in $20 \mathrm{seg}$ ments and dividing the flux in each by its mean value. This algorithm is very robust to changes in the signal-to-noise ratio, and our own simulations show that has excellent performance.
Previous high-resolution spectroscopic studies of these stars have shown that they are associated with the CEMP-no class (carbon-enhanced metal-poor stars without over-abundances of neutron-capture elements; see, e.g., Beers \& Christlieb 2005; Hansen et al. 2014). The relatively low effective temperature of this $\operatorname{star}\left(T_{\text {eff }} \sim 5400 \mathrm{~K}\right)$ and the high signal-to-noise of his BOSS spectrum, allows us to determine the abundances of carbon, calcium and iron, despite the fairly low spectral resolution $(\lambda / \delta \lambda \sim 2000)$. From our estimated calcium abundance $[\mathrm{Ca} / \mathrm{H}] \simeq-4.0$, and iron abundance $[\mathrm{Fe} / \mathrm{H}] \simeq-4.3$, J1313-0019 is among the ten most iron-poor stars known.

Figure 3 shows the $\mathrm{C} / \mathrm{Fe}$ ratio for the stars with the lowest iron abundances known. In the case of SMSS J031300.36670839.3 (Keller et al. 2014), only an upper limit to the iron abundance is available $([\mathrm{Fe} / \mathrm{H}]<-7.1)$. The sample of known metal-poor stars dramatically increases at $[\mathrm{Fe} / \mathrm{H}]>-4$.
Chemical evolution models predict that the transition between a regime dominated by stochastic star-formation events, where the gas is polluted by a single or a few supernovae, and a well-mixed interstellar gas where the instantaneous mixing approximation works, takes place at about $[\mathrm{Fe} / \mathrm{H}] \sim-4$ (Audouze \& Silk 1995; Oey 2003; Karlsson 2005).

We focus our attention on the 13 stars known with $[\mathrm{Fe} / \mathrm{H}]<-4$. The parameters for these stars are drawn from those derived or compiled by Caffau et al. (2012), Norris et al. (2013), Keller et al. (2014), and Hansen et al. (2014). About half of the sample, including J1313-0019, have a very large carbon enrichment $[\mathrm{C} / \mathrm{Fe}]>+2$, while the others exhibit a lower $\mathrm{C} / \mathrm{Fe}$ ratio, but still supersolar. The most carbon-rich stars show that the $\mathrm{C} / \mathrm{Fe}$ ratio is anticorrelated with the iron abundance. This implies that, as suggested by Spite et al. (2013), the carbon abundance in these stars is $[\mathrm{C} / \mathrm{H}] \sim-1.5$, irrespective of $[\mathrm{Fe} / \mathrm{H}]$. This carbon 


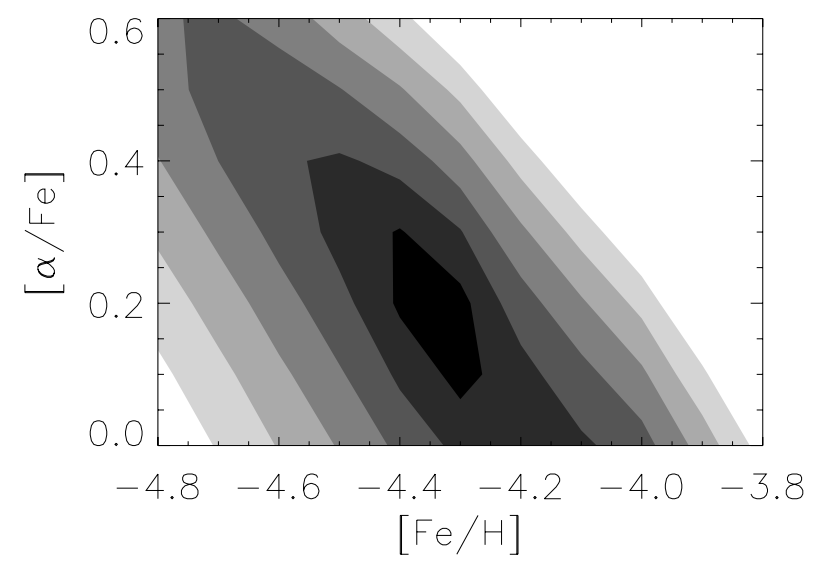

Fig. 2. Contours of the $\chi^{2}$ statistic in the $[\mathrm{Fe} / \mathrm{H}]-[\alpha / \mathrm{Fe}]$ plane, corresponding to $1-2-3$...etc $\sigma$ uncertainties in these parameters.

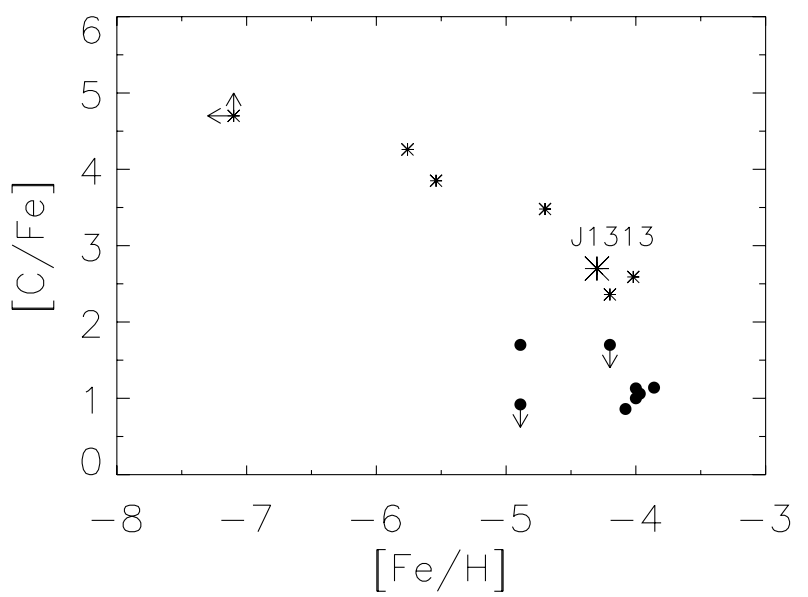

Fig. 3. Carbon-to-iron ratio vs. iron abundance in stars with $[\mathrm{Fe} / \mathrm{H}]<$ -4 . Asterisks are used for those with $[\mathrm{C} / \mathrm{Fe}]>+2$, and filled circles for those with $[\mathrm{C} / \mathrm{Fe}]<+2$. A larger symbol identifies the newly identified star J1313-0019.

abundance is well above the threshold for line cooling that facilitates the gas cloud fragmentation required to form low-mass stars (Frebel et al. 2009).

The source of carbon for ultra metal-poor stars is hydrostatic helium burning in high-mass zero-metallicity (population III) stars that end their lives as supernovae. The amount of carbon released in each event is a fairly sensitive function of the mixing and fallback of material in the supernovae, which is difficult to model in detail. Keller et al. have recently argued that the abundance pattern in SMSS J031300.36 - 670839.3 could be created by a single supernova with a mass of the progenitor metal-free star in the vicinity of $60 M_{\odot}$. We have explored yields from models by Heger \& Woosley (2010), and as shown in the upper panel of Fig. 4, there are multiple combinations of the progenitor mass and the explosion energy that lead to $\mathrm{C} / \mathrm{Fe}$ mass ratios in the range found in the carbon-rich ultra iron-poor stars discussed in this paper. These models consider non-rotating stars with masses between 10 and $100 M_{\odot}$, and follow their evolution, and final supernova explosions. We note that there are several other scenarios for the production of the elemental abundances found in carbon-rich ultra metal-poor stars (see, e.g. Maeder et al. 2015), but we examine these models in detail since they have already been used in previous studies of similar stars, and they are provided in a format that can be readily used for comparison with observations. Metal-free stars with masses between
140-260 $M_{\odot}$ end their lives as pair-instability supernovae, but calculations by Heger \& Woosley (2002) show that their carbonto-iron ratios are close to solar, with the exception of the stars in the lowest end of that range, whose yields can reach up to a mass fraction $\mathrm{C} / \mathrm{Fe} \sim 10^{10}$.

The lower panel of Fig. 4 shows the log of the ratio of the carbon and iron yields, against the log of the iron yield in solar masses, again following calculations by Heger \& Woosely (2010). The ejecta from the supernovae will be diluted in the interstellar gas, but the carbon-to-iron ratio should in principle be preserved. After changing from mass fraction to particle fraction, we have subtracted 0.94 , the log of the solar ratio of carbon and iron abundances, to the supernova yields before placing them in the figure $([\mathrm{C} / \mathrm{Fe}]=\log (\mathrm{C} / \mathrm{Fe})+\log (56 / 12)-0.94)$. The iron-to-hydrogen ratio in the interstellar medium (and in the atmospheres of these primitive stars) can be arbitrarily shifted from the supernova yields in solar masses to make the horizontal axis in the figure, and we have shifted the log of the iron yield in solar masses by -0.7 dex in order to match the observations.

There is a fairly tight relationship between the carbon-to-iron ratio and the iron yields for these supernovae. For some models the carbon-to-iron ratios are much larger, up to $[\mathrm{C} / \mathrm{Fe}]$ of about 20 and out of the range shown in the figure, but few models predict lower ratios, especially for high values of the iron yields. All of the stars with $[\mathrm{Fe} / \mathrm{H}]<-4$ and $[\mathrm{C} / \mathrm{Fe}]>+2$ (asterisks in Fig. 4), including J1313-0019, match the trend predicted by theory, suggesting that those stars could be formed out of gas polluted by supernovae resulting from metal-free stars in the range 10-100 $M_{\odot}$. This correlation is not observed for the ultra metalpoor stars that show $[\mathrm{C} / \mathrm{Fe}]<+2$, indicating that their chemical composition is associated with a different type of source, that the models considered do not span a sufficiently large range in explosion energy, or a systematic effect exists due to rotation (missing in the models). Interestingly, neither the iron yields, nor the carbon-to-iron ratios of the models correlate with the mass of the progenitor. There also does not exist a correlation of the abundances with the energy of the explosion.

Our result of supernovae from Population III stars matching the compositions of ultra iron-poor carbon-rich stars is in line with previous investigations that found that models can reproduce in a fairly good detail the chemical patterns measured in such stars (e.g., Joggerst et al. 2010; Keller et al. 2014). One of our most surprising findings is that we are able to measure several elemental abundances for ultra-low metallicity stars from low-resolution spectroscopy. The literature is polarized between studies at high spectral resolution and high signal-to-noise ratios, and those at a modest resolution with limited signal-to-noise ratios. However, high signal-to-noise observations at limited resolution seem to provide a promising avenue for making significant advances in this field. The Sloan Digital Sky Survey continues to obtain observations of stars at high Galactic lattitude, and to identify chemically primitive stars. Building a larger sample of such rare objects will be critical for understanding the formation and early chemical evolution of galaxies, and in particular the Milky Way.

Acknowledgements. T.C.B. acknowledges partial support for this work from grants PHY 08-22648: Physics Frontier Center/Joint Institute for Nuclear Astrophysics (JINA), and PHY 14-30152: Physics Frontier Center/JINA Center for the Evolution of the Elements (JINA-CEE), awarded by the US National Science Foundation. C.A.P., D.S.A., J.I.G.H., and R.R. are thankful for support by the Spanish Ministry of Economy and Competitiveness under grant AYA2014-56359-P. Funding for SDSS-III has been provided by the Alfred P. Sloan Foundation, the Participating Institutions, the National Science Foundation, and the US Department of Energy Office of Science. 

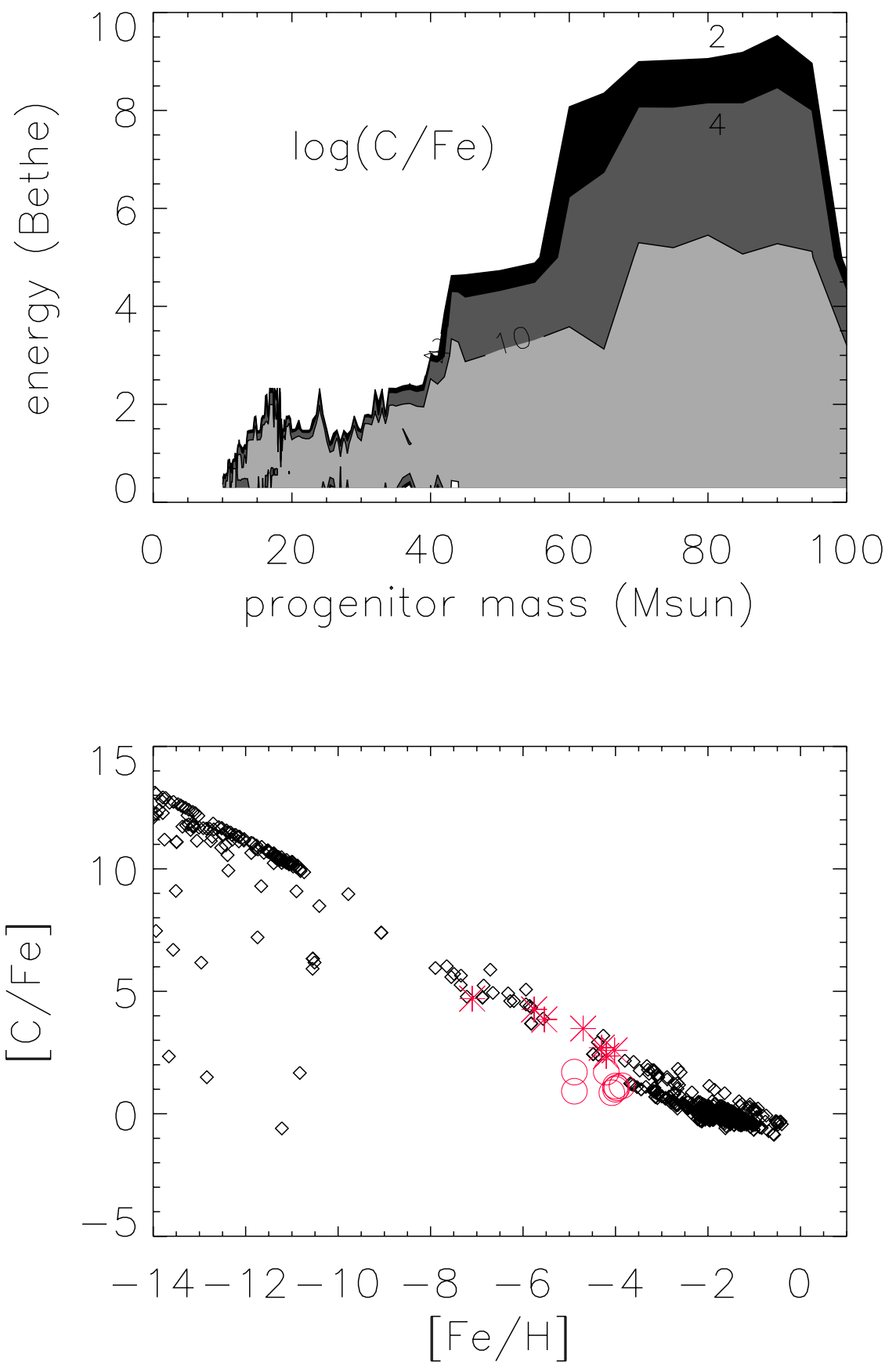

Fig. 4. Top panel: contour diagram showing the carbon-to-iron mass ratio, as a function of progenitor mass and explosion energy, predicted for the supernovae yields of zero metallicity stars by Heger \& Woosley (2010). Contours are shown for $\log (\mathrm{C} / \mathrm{Fe})=2,4,10$ and 20. Bottom panel: the carbon-to-iron ratio, as a function of iron production, predicted for the supernovae models (rhombi; with iron multiplied by an arbitrary factor), and abundances found in the most ironpoor stars known (the upper limits for iron in SMSSJ031300.36-670839.3 have been ignored for simplicity). The asterisks correspond to stars that have $[\mathrm{C} / \mathrm{Fe}]>+2$, and the open circles show those with lower carbon-to-iron ratios.
The SDSS-III web site is http://www.sdss3.org/. SDSS-III is managed by the Astrophysical Research Consortium for the Participating Institutions of the SDSS-III Collaboration including the University of Arizona, the Brazilian Participation Group, Brookhaven National Laboratory, Carnegie Mellon University, University of Florida, the French Participation Group, the German Participation Group, Harvard University, the Instituto de Astrofisica de Canarias, the Michigan State/Notre Dame/JINA Participation Group, Johns Hopkins University, Lawrence Berkeley National Laboratory, Max Planck Institute for Astrophysics, Max Planck Institute for Extraterrestrial Physics, New Mexico State University, New York University, Ohio State University, Pennsylvania State University, University of Portsmouth, Princeton University, the Spanish Participation Group, University of Tokyo, University of Utah, Vanderbilt University, University of Virginia, University of Washington, and Yale University.

\section{References}

Abel, T., Bryan, G. L., \& Norman, M. L. 2002, Science, 295, 93 Ahn, C. P., Alexandroff, R., Allende Prieto, C., et al. 2012, ApJS, 203, 21
Alam, S., Albareti, F. D., Allende Prieto, C., et al. 2015, ApJS, submitted [arXiv: 1501.00963]

Allende Prieto, C., Beers, T. C., Wilhelm, R., et al. 2006, ApJ, 636, 804

Allende Prieto, C., Fernández-Alvar, E., Schlesinger, K. J., et al. 2014, A\&A 568, A7

Audouze, J., \& Silk, J. 1995, ApJ, 451, L49

Beers, T. C., \& Christlieb, N. 2005, ARA\&A, 43, 531

Bolton, A. S., Schlegel, D. J., Aubourg, É., et al. 2012, AJ, 144, 144

Bromm, V., \& Loeb, A. 2003, Nature, 425, 812

Bromm, V., Coppi, P. S., \& Larson, R. B. 1999, ApJ, 527, L5

Caffau, E., Bonifacio, P., François, P., et al. 2011, Nature, 477, 67

Caffau, E., Bonifacio, P., François, P., et al. 2012, A\&A, 542, A51

Christlieb, N., Bessell, M. S., Beers, T. C., et al. 2002, Nature, 419, 904

Clark, P. C., Glover, S. C. O., Smith, R. J., et al. 2011, Science, 331, 1040

Dawson, K., Schlegel, D. J., Ahn, C. P., et al. 2013, AJ, 145, 10

Eisenstein, D. J., Weinberg, D. H., Agol, E., et al. 2011, AJ, 142, 72

Fernández-Alvar, E., Allende Prieto, C., Schlesinger, K. J., et al. 2015, A\&A, 577, A81

Frebel, A., Aoki, W., Christlieb, N., et al. 2005, Nature, 434, 871

Frebel, A., Johnson, J. L., \& Bromm, V. 2009, MNRAS, 392, L50 
A\&A 579, A98 (2015)

Girardi, L., Grebel, E. K., Odenkirchen, M., \& Chiosi, C. 2004, A\&A, 422, 205 Greif, T. H., Springel, V., White, S. D. M., et al. 2011, ApJ, 737, 75

Gunn, J. E., Siegmund, W. A., Mannery, E. J., et al. 2006, AJ, 131, 2332

Hansen, T., Hansen, C. J., Christlieb, N., et al. 2014, ApJ, 787, 162

Heger, A., \& Woosley, S. E. 2002, ApJ, 567, 532

Heger, A., \& Woosley, S. E. 2010, ApJ, 724, 341

Ji, A. P., Frebel, A., \& Bromm, V. 2014, ApJ, 782, 95

Joggerst, C. C., Almgren, A., Bell, J., et al. 2010, ApJ, 709, 11

Karlsson, T. 2005, A\&A, 439, 93

Keller, S. C., Bessell, M. S., Frebel, A., et al. 2014, Nature, 506, 463

Lee, Y. S., Beers, T. C., Sivarani, T., et al. 2008, AJ, 136, 2022

Maeder, A., Meynet, G., \& Chiappini, C. 2015, A\&A, 576, A56
Mészáros, S., Allende Prieto, C., Edvardsson, B., et al. 2012, AJ, 144, 120 Norris, J. E., Christlieb, N., Korn, A. J., et al. 2007, ApJ, 670, 774

Norris, J. E., Yong, D., Bessell, M. S., et al. 2013, ApJ, 762, 28

Oey, M. S. 2003, MNRAS, 339, 849

Pinsonneault, M. H., An, D., Molenda-Żakowicz, J., et al. 2012, ApJS, 199, 30

Schneider, R., Omukai, K., Limongi, M., et al. 2012, MNRAS, 423, L60

Silk, J. 1997, ApJ, 481, 703

Smee, S. A., Gunn, J. E., Uomoto, A., et al. 2013, AJ, 146, 32

Sneden, C. A. 1973, in Ph.D. Thesis, The University of Texas at Austin, 35

Spite, M., Caffau, E., Bonifacio, P., et al. 2013, A\&A, 44, 107

Truran, J. W., \& Cameron, A. G. W. 1971, Ap\&SS, 14, 179

Yanny, B., Rockosi, C., Newberg, H. J., et al. 2009, AJ, 137, 4377 